\title{
Risk factor assessment in South African Black patients presenting with acute myocardial infarction at R.K. Khan Hospital, Durban
}

\section{S.C. Masina*, N. Ranjith" and B. Sartorius'}

*Department of Medicine, Nelson R. Mandela School of Medicine, University of KwaZulu-Natal, Durban, South Africa

"Department of Medicine, Coronary Care Unit, R.K. Khan Hospital, Durban, South Africa

tDepartment of Public Health Medicine, School of Nursing and Public Health, University of KwaZulu-Natal, Durban, South Africa,

Address for correspondence:

Prof N. Ranjith

R.K. Khan Hospital

Department of Medicine

Coronary Care Unit

Private Bag X004

Chatsworth

Durban

4030

South Africa

Email:

ranjith@lantic.co.za

\section{INTRODUCTION}

Coronary Artery Disease (CAD) is a major cause of death and disability, worldwide. Similarly, in South Africa, which is a multiethnic society with a large range of cultures and life styles, CAD is also a major cause of morbidity and mortality, particularly amongst the White and Indian populations. ${ }^{(1-3)}$ In contrast, earlier studies found that CAD was relatively uncommon in the Black population, and this low incidence was suggested to be due to lower serum cholesterol and increased high-density lipoprotein (HDL) cholesterol levels. ${ }^{(4)}$ In addition, assessment of selected risk factors for CAD in young South African male scholars by Seftel, et al. showed that both Indian and White scholars had a much higher prevalence and severity of CAD risk factors than Black youths. ${ }^{(5)}$

However, recently the INTERHEART Africa study reported that more premature acute myocardial infarctions (AMI) occur in sub-saharan Africa than in any of the other 52 countries participating in the INTERHEART study. ${ }^{(6,7)}$ This is due largely to the modeling of 5 risk factors (smoking, diabetes, hypertension, abdominal obesity and dyslipidaemia), which provide a population attributable risk of $89.2 \%$ for AMI. These results are consistent with the overall INTERHEART Study. Of note is that the prevalence of CAD is also increasing in the South African Black population because they are exposed to a similar risk

\section{ABSTRACT}

Objective: To assess various risk factors in Black South African patients presenting with acute myocardial infarction (AMI) to the Coronary Care Unit (CCU) at R.K. Khan Hospital, Durban, South Africa.

Methods and results:The study population was recruited from consecutive patients from a multi-ethnic background with a diagnosis of AMI over a 4 year period (2008 - 20I2). Only patients who were of Black South African origin $(n=94)$ were eligible for analysis. Demographic data stored in an electronic database were extracted from all patients. The mean age of the participants was $54.3 \pm 1 \mathrm{I} .0$ years of whom $85 \%$ were males. The majority presented with ST elevation myocardial infarction (STEMI) (83\%) whilst I7\% had Non ST elevation myocardial infarction (NSTEMI). Smoking (48\%) and hypertension (46\%) were the most commonly observed risk factors. Thirty six percent of patients developed one or more major adverse cardiac events (MACE). Recurrence of infarction and death occurred more frequently in females compared to males (14\% vs. $2 \%, p=0.04$ ).

No significant difference in MACE was found between patients who were human immunodeficiency virus (HIV) positive (18\%) compared to those who were HIV negative $(82 \%)$. Metabolic syndrome, as defined by the International Diabetes Federation (IDF) criteria, was found in $35(45 \%)$ of patients. The number of patients presenting with hypertension and diabetes was significantly higher in our study compared to the INTERHEART Africa study ( $73 \%$ vs. $50 \%, p<0.01$; and $40 \%$ vs. $24 \%, p<0.01$ ).

Conclusion: South African Blacks have multiple risk factors, similar to other ethnic groups in the country, which contribute to the development of AMI. Recurrence of infarction and death occurred more frequently in females compared to males. In addition to conventional risk factors for CAD, an increased heart rate, STEMI, obesity, family history of cerebrovascular disease and hyperuricaemia are strongly associated with the presence of MACE. SAHeart 2016;13:12-18

profile, due to greater urbanisation and the adoption of an unhealthy life style. ${ }^{(8)}$

Another important contributor to the increase in CAD in this ethnic group may be related to the human immunodeficiency virus (HIV), as South Africa is the country with the highest number of HIV infections in the world. ${ }^{(9)}$ It has been suggested that HIV infection per se should count as a coronary risk factor, 
similar to the traditional cardiovascular risk factors, since HIV infections may promote early atherosclerosis independently of these classical vascular risk factors. ${ }^{(10)}$

The prevalence rate of the metabolic syndrome is also increasing in Black South Africans, which could be another factor contributing to their increase in CAD. ${ }^{(11)}$ Despite the growing burden of CAD in the Black population, there is still a paucity of information concerning the association between CAD and its known risk factors. The primary objective of this study, therefore, was to assess various risk factors in Black South African patients presenting with AMI to the Coronary Care Unit (CCU) at R.K. Khan Hospital, Durban.

\section{METHODS}

The study population was recruited from consecutive patients from a multi-ethnic background, with a diagnosis of AMI within 24 hours of onset of symptoms to the $\mathrm{CCU}$ at R.K. Khan Hospital, Durban, over a four year period (2008 - 20 I2). Of the I 200 patients admitted with AMI, 87\% were of Asian Indian origin, $7.8 \%$ Blacks, $4.4 \%$ Whites, and $0.8 \%$ Coloureds. Only patients of South African Black origin $(n=94)$ were eligible for analysis. The study was carried out according to the principles of the Declaration of Helsinki and was approved by the local ethics committee. Acute myocardial infarction was defined by prolonged chest discomfort, typical electrocardiographic (ECG) changes, and elevated cardiac troponin $\mathrm{T}$ (cTnT) levels as outlined by the Joint European Society of Cardiology/American College of Cardiology Committee. ${ }^{(12)}$

\section{Clinical assessment}

Demographic data stored in an electronic database were extracted for all eligible patients. Every patient underwent an initial assessment that included clinical history, physical examination, and 12-lead surface ECG. Current smokers were defined as those individuals who had smoked any tobacco in the previous 12 months, and former smokers as those who had not smoked for a period of at least a year. A family history of premature atherosclerosis was defined by a history of myocardial infarction in either parents, or in siblings, or first degree relatives at the age of 55 years or younger, for males, and 65 years or younger, in females, whilst a family history of diabetes, hypertension, and cerebrovascular disease was defined as these conditions occurring at any age. Additional clinical data included a detailed description of major adverse cardiac events (MACE) encountered during hospital admission until 6 months followup, such as ventricular arrhythmias, complete heart block, cardiac failure, cardiogenic shock, recurrence of angina or myocardial infarction and death.

In keeping with standard acute coronary syndrome guidelines, all patients were counseled and offered coronary angiograms, which were performed at another referral hospital. Patients were required to consent to both coronary angiography and cardiac revascularisation procedures such as percutaneous coronary intervention $(\mathrm{PCl})$ and coronary artery bypass grafting
( $C A B G$ ), should there be a need to perform these procedures. This type of triage is necessary due to the large volume of work and the lack of adequate facilities in the public sector (only I government-based hospital in the province of KwaZulu-Natal offers these specialised facilities). Consequently, only a limited number of patients underwent coronary angiography because the majority declined cardiac revascularisation. Patients who were eligible were thrombolysed with metalyse as a reperfusion therapy.

Since the INTERHEART Africa Study is probably the most comprehensive case-control study conducted among patients with AMI in sub-saharan Africa (SSA), we also explored the association of known cardiovascular risk factors and AMI between the Black African group of this study $(n=144)$ and our study population.

\section{Anthropometric measurements}

The body mass index (BMI) was calculated as weight (kilograms) divided by height ${ }^{2}$ (meters) according to the World Health Organisation guidelines. ${ }^{(13)}$

A BMl $\geq 30 \mathrm{~kg} / \mathrm{m}^{2}$ was used to define adult obesity. Waist circumference, which is considered the most practical way to assess central obesity, was measured midway between the lowest rib and the iliac crest on standing subjects, using a soft tape. The presence of the metabolic syndrome, as outlined by the International Diabetes Federation (IDF), was defined as central obesity (waist circumference: males $\geq 94 \mathrm{~cm}$, females $\geq 80 \mathrm{~cm}$ ) plus any 2 of the criteria shown in Table $2^{\left({ }^{(14)}\right.}$

\section{Biochemical Analyses}

Blood samples for routine biochemistry were collected from all subjects on hospital admission and carried out using standard methods. Troponin T measured on the Elecsys 2010 (Roche Diagnostics) was considered positive at a cut-off value greater than $0.03 \mathrm{ng} / \mathrm{ml}$. Blood samples were also collected within 48 hours of admission after an overnight fast for total cholesterol, triglycerides and HDL cholesterol, which were measured on the Beckman Synchron CX7 auto analyser. Low-density lipoprotein (LDL) cholesterol levels were calculated utilising the formula of Friedewald. The HIV status of every patient was determined using the Enzyme linked immunosorbent assay (ELISA).

\section{Statistical analyses}

Data were processed and analysed using Stata 13.0 (StataCorp. 20 I3. Stata Statistical Software: Release 13. College Station, TX: StataCorp LP). Differences in means of continuous variables by dichotomous classification (e.g. gender, HIV status and adverse cardiac events) were assessed using t-tests (or the non-para-metric equivalent, namely the Wilcoxon Rank Sum Test). Differences in frequencies of categorical demographic variable by metabolic syndrome status and other categorical outcomes were assessed using the Pearson chi-square $(\chi 2)$ test. If an expected cell count has fewer than 5 observations, then the Fishers Exact Test P-values are presented instead. 
Bivariate and multivariable logistic regression was employed to estimate the association between clinical and laboratory parameters and MACE. Coefficients were exponentiated to represent odds ratios (ORs) and $95 \%$ confidence intervals. An adjusted $\mathrm{P}$-value of $<0.05$ was deemed statistically significant.

\section{RESULTS}

Of the 94 patients in the study cohort, 58 (85\%) were males. The mean age of the study population was $54.3 \pm 11.0$ years, with the majority presenting with ST elevation myocardial infarction (STEMI) (83\%), whilst 17\% had Non ST elevation myocardial infarction (NSTEMI) (Table I). Previous and current smoking (48\%) and hypertension (46\%) were the most commonly observed risk factors. A significantly greater number of men had a history of smoking compared to their female counterparts [72\% vs. 8\%, OR 28.87 (95\% Cl 7.75 - 107.52); $\mathrm{p}<0.0 \mathrm{I}$ ], while females in contrast were found to have a higher incidence of hypertension [67\% vs. 33\%, OR $0.24(95 \% \mathrm{Cl}$ 0.1 - 0.59); $p<0.01$ ]. Although haemoglobin levels were significantly lower in female patients $(12.38 \pm 1.91 \mathrm{~g} / \mathrm{dl}$ vs. $14.06 \pm$ $1.9 \mathrm{~g} / \mathrm{dl}, \mathrm{p}<0.01)$, male patients had higher creatinine values ( $01.24 \pm 27.85$ umol/L vs. $83.08 \pm 21.3$ umol/L, $p<0.0$ l).

From index hospitalisation until 6-month follow-up, 36\% of patients developed one or more MACE. Recurrence of infarction and death occurred more frequently in females compared to males [I $4 \%$ vs. $2 \%$, OR 0.1 I (95\% Cl 0.01 - 0.97); $p=0.04]$. No significant difference was found in traditional cardiovascular risk factors and MACE between patients who were HIV positive ( I $8 \%$ ) compared to those who were HIV negative $(82 \%$, data not shown). Only 23 patients had coronary angiograms performed at another referral hospital because most patients declined coronary revascularisation. Thirty five percent presented with triple vessel disease, $22 \%$ with double vessel, and $26 \%$ with single vessel disease while $17 \%$ had normal coronary angiograms.

Metabolic syndrome, as defined by the IDF criteria, was found in 35 (45\%) of the study population (Table 2). Central obesity was the major IDF determinant in patients with or without the metabolic syndrome ( $100 \%$ vs. 61\%, $p<0.01)$, while elevated blood pressure (83\%), low HDL cholesterol levels (80\%), raised triglyceride (48\%), and fasting blood glucose values (46\%) occurred significantly more frequently in patients with the metabolic syndrome.

Table 3 compares the risk profile for AMI between the Black population of the INTERHEART Africa study and the R.K. Khan Hospital study. The number of patients presenting with hypertension and diabetes was significantly higher in our study compared to the INTERHEART Africa study (73\% vs. 50\%, p<0.0।; and $40 \%$ vs. $24 \%, p<0.01$ ), respectively. The lipid profiles were similar for the 2 study groups.

Figure I illustrates the presence of various combinations of any 6 risk factors for AMI, namely diabetes, smoking, family history of vascular disease, dyslipidaemia, hypertension and central obesity. Of note is that $32 \%$ of patients had 4 risk factors, $20 \%$ presented with 5 risk factors, and overall $89 \%$ had 2 or more risk factors for AMI.

A multivariable analysis for factors associated with MACE by logistic regression was done (Table 4). After multivariable adjustment, an increased heart rate ( $>100$ beats per minute) (OR I.06 [95\% Cl I.0 I - I. I0]; p=0.0 I), STEMI (OR 5.89 [95\% Cl 1.22 - 28.5I]; $p=0.03$ ), obesity (OR $3.86[95 \% \mathrm{Cl} 1.18$ 12.63]; $p=0.03$ ), a family history of cerebrovascular disease (OR | 4.83 [95\% Cl I. 10 - 200.82]; $p=0.04$ ) and hyperuricaemia (OR 3.64 [95\% Cl I.05 - 12.66]; $p=0.04$ ) was significantly associated with MACE.

\section{DISCUSSION}

In this study, South African Black patients were characterised by the presence of multiple risk factors for AMI. Previous and current smoking (48\%), hypertension (46\%), and diabetes (40\%) were the most commonly observed risk factors. Of note is that $89 \%$ of patients had 2 or more risk factors for $\mathrm{AMI}$, with $32 \%$ presenting with 4 risk factors while $20 \%$ had 5 risk factors. These results are consistent with the INTERHEART Africa Study and confirm that people from Africa, particularly Black South Africans who, when exposed to known major CAD risk factors, are at risk to develop AMI, as are people across the globe. ${ }^{(15)}$

Furthermore, our data show that patients in the R.K. Khan Hospital study had a significantly higher prevalence of hypertension $(p<0.0 \mathrm{I})$ and diabetes $(\mathrm{p}<0.0 \mathrm{I})$ compared to the Black patients in the INTERHEART Africa study. These results assume greater importance since it was reported that a history of hypertension was significantly stronger in the total African population compared to the global INTERHEART population ( $p=0.002$ for tests of heterogeneity of effects). ${ }^{(6)}$ What was once considered a rare disease in this ethnic group, ${ }^{(16,17)}$ is now observed more frequently, suggesting that uncontrolled major CAD risk factors will have a larger impact on the burden of CAD in Africa than elsewhere. This is supported by the fact that although only $25 \%$ of patients in the study cohort underwent cardiac catheterisation studies, most patients presented with advanced triple vessel disease (35\%), with 17\% having normal coronary angiograms. In addition, $36 \%$ of subjects experienced MACE during the study follow-up period, with recurrence of myocardial infarction $(p=0.04)$ and death $(p=0.04)$ occurring more often in females compared to their male counterparts.

Several studies have reported an association between HIV disease and CAD, which may occur because of dyslipidaemia, glucose intolerance and endothelial dysfunction secondary to some antiretroviral drugs. ${ }^{(18,19)}$ This is of particular importance in the South African setting, since it is the country with the highest number of HIV infections in the world. ${ }^{(9)}$ Eighteen percent of the study population were found to be HIV positive but none 
TABLE I: Baseline characteristics and gender differences.

\begin{tabular}{|c|c|c|c|c|c|}
\hline Characteristics & $\begin{array}{l}\text { All patients \% } \\
(n=94)\end{array}$ & $\begin{array}{l}\text { Males \% } \\
(n=58)\end{array}$ & $\begin{array}{c}\text { Females \% } \\
(n=36)\end{array}$ & $\begin{array}{c}\text { OR }(95 \% \mathrm{Cl}) \\
\text { Males vs. Females }\end{array}$ & P-value \\
\hline STEMI & 83 & 85 & 81 & \multirow{2}{*}{$1.31(0.44-3.91)$} & \multirow{2}{*}{0.62} \\
\hline NSTEMI & 17 & 15 & 19 & & \\
\hline \multicolumn{6}{|l|}{ Body mass index } \\
\hline $\operatorname{Normal}\left(\leq 25 \mathrm{~kg} / \mathrm{m}^{2}\right)$ & 26 & 38 & 6 & $10.39(2.27-47.57)$ & $<0.01$ \\
\hline Pre-obese $\left(26-29 \mathrm{~kg} / \mathrm{m}^{2}\right)$ & 35 & 36 & 33 & $1.14(0.47-2.73)$ & 0.78 \\
\hline Obese $\left(\geq 30 \mathrm{~kg} / \mathrm{m}^{2}\right)$ & 31 & 24 & 42 & $0.45(0.18-1.09)$ & 0.08 \\
\hline \multicolumn{6}{|l|}{ Risk Factors } \\
\hline Previous and current smoking & 48 & 72 & 8 & $28.87(7.75-107.52)$ & $<0.01$ \\
\hline Hypertension & 46 & 33 & 67 & $0.24(0.1-0.59)$ & $<0.01$ \\
\hline Diabetes & 40 & 33 & 53 & $0.44(0.19-1.02)$ & 0.06 \\
\hline Previous angina & 5 & 5 & 6 & $0.93(0.15-5.84)$ & 0.94 \\
\hline Previous myocardial infarction & 4 & 5 & 3 & $1.91(0.19-19.09)$ & 0.58 \\
\hline \multicolumn{6}{|c|}{ Family History of vascular disease } \\
\hline Hypertension & 18 & 16 & 22 & $0.64(0.22-1.85)$ & 0.41 \\
\hline Diabetes & 17 & 12 & 25 & $0.41(0.14-1.23)$ & 0.11 \\
\hline Coronary Artery disease & 9 & 7 & 11 & $0.59(0.14-2.53)$ & 0.48 \\
\hline Cerebrovascular disease & 5 & 4 & 8 & $0.39(0.06-2.47)$ & 0.32 \\
\hline Continuous variables & Mean (SD) & Mean (SD) & Mean (SD) & $\begin{array}{c}\text { OR }(95 \% \mathrm{Cl}) \\
\text { Males vs. Females }\end{array}$ & P-value \\
\hline Age (years) & $54.3(11.0)$ & $53.02(9.9)$ & $56.31(12.37)$ & $0.97(0.93-1.01)$ & 0.16 \\
\hline Systolic blood pressure $(\mathrm{mmHg})$ & $142.29(29.04)$ & $138.03(26.4)$ & $149.14(32.05)$ & $0.99(0.97-1.0)$ & 0.08 \\
\hline Diastolic blood pressure $(\mathrm{mmHg})$ & $88.33(20.56)$ & $87.31(19.01)$ & $89.97(23.04)$ & $0.99(0.97-1.01)$ & 0.54 \\
\hline Abdominal girth $(\mathrm{cm})$ & $99.88(13.5)$ & $97.98(14.27)$ & $102.94(11.72)$ & $0.97(0.94-1.01)$ & 0.11 \\
\hline \multicolumn{6}{|l|}{ Biochemical Data } \\
\hline Haemoglobin (g/dl) & 13.42(2.06) & 14.06 (1.9) & $12.38(1.91)$ & $1.66(1.25-2.2)$ & $<0.01$ \\
\hline Blood glucose (mmol/L) & $8.87(5.76)$ & $8.22(5.11)$ & $9.92(6.61)$ & $0.95(0.88-1.02)$ & 0.17 \\
\hline Creatinine (umol/L) & $94.29(26.96)$ & $101.24(27.85)$ & $83.08(21.3)$ & $1.04(1.01-1.07)$ & $<0.01$ \\
\hline Uric acid (mmol/L) & $0.39(0.13)$ & $0.39(0.14)$ & $0.39(0.12)$ & $1.03(0.04-25.96)$ & 0.99 \\
\hline Total cholesterol (mmol/L) & $4.8(1.74)$ & $4.77(1.86)$ & $4.85(1.54)$ & $0.97(0.77-1.23)$ & 0.82 \\
\hline LDL cholesterol (mmol/L) & $3.20(1.12)$ & $2.9(1.01)$ & $3.22(1.28)$ & $0.77(0.52-1.15)$ & 0.20 \\
\hline HDL cholesterol (mmol/L) & $1.30(0.35)$ & $1.01(0.34)$ & $1.05(0.37)$ & $0.7 I(0.21-2.4 I)$ & 0.58 \\
\hline Triglycerides (mmol/L) & $1.48(1.4 \mathrm{I})$ & $1.58(1.63)$ & $1.32(0.97)$ & $1.17(0.81-1.69)$ & 0.40 \\
\hline MACE & 36 & 31 & 44 & $0.56(0.24-1.33)$ & 0.19 \\
\hline Cardiac failure & 18 & 14 & 25 & $0.48(0.17-1.39)$ & 0.18 \\
\hline Death & 6 & 2 & 14 & $0.11(0.01-0.97)$ & 0.04 \\
\hline Recurrence of infarction & 6 & 2 & 14 & $0.11(0.01-0.97)$ & 0.04 \\
\hline Ventricular arrhythmia & 6 & 9 & 3 & $3.3(0.37-29.48)$ & 0.29 \\
\hline Atrial fibrillation & 4 & 3 & 6 & $0.61(0.08-4.51)$ & 0.63 \\
\hline Cardiogenic shock & 4 & 5 & 3 & $1.91(0.19-19.09)$ & 0.58 \\
\hline Recurrence of angina & 3 & 2 & 6 & $0.3(0.03-3.4 I)$ & 0.33 \\
\hline Cerebrovascular accident & 3 & 3 & 3 & $1.25(0.11-14.3)$ & 0.86 \\
\hline Heart block & 2 & 3 & 0 & NC & 0.52 \\
\hline \multicolumn{6}{|l|}{ Medication at discharge } \\
\hline Nitrates & 100 & 100 & 100 & NC & \\
\hline Disprin & 100 & 100 & 100 & NC & \\
\hline Statins & 94 & 93 & 94 & $0.79(0.14-4.57)$ & 0.80 \\
\hline ACEI/ARB & 89 & 86 & 94 & $0.37(0.07-1.84)$ & 0.22 \\
\hline Beta blockers & 56 & 55 & 58 & $0.88(0.38-2.04)$ & 0.76 \\
\hline Calcium antagonist & 6 & 5 & 8 & $0.60(0.11-3.15)$ & 0.55 \\
\hline Coronary Angiogram & $n=23$ & $n=14$ & $n=9$ & \multirow{4}{*}{$1.61(0.35-4.48)$} & \multirow{4}{*}{0.54} \\
\hline Single vessel disease & 26 & 21 & 33 & & \\
\hline Double vessel disease & 22 & 29 & 11 & & \\
\hline Triple vessel disease & 35 & 36 & 33 & & \\
\hline CABG & 17 & 14 & 22 & $0.58(0.07-5.11)$ & 0.63 \\
\hline $\mathrm{PCl}$ & 0 & 0 & 0 & NC & \\
\hline
\end{tabular}

MACE $=$ Major Adverse Cardiac Events, $N C=$ Not Calculated 
TABLE 2: Prevalence of the metabolic syndrome according to the IDF definition and frequency of individual criteria.

\begin{tabular}{|c|c|c|c|c|}
\hline IDF Criteria & $\begin{array}{l}\text { All patients } \\
\%(n=8 I)\end{array}$ & $\begin{array}{l}\text { Patients with Metabolic } \\
\text { Syndrome \% }(n=35)\end{array}$ & $\begin{array}{l}\text { Patients without } \\
\text { Metabolic Syndrome \% } \\
(n=46)\end{array}$ & P-value \\
\hline $\begin{array}{l}\text { Waist circumference } \\
\text { (male } \geq 94 \mathrm{~cm} \text {, females } \geq 80 \mathrm{~cm} \text { ) }\end{array}$ & 78 & 100 & 61 & $<0.01$ \\
\hline $\begin{array}{l}\text { Fasting glucose } \\
\geq 5.6 \mathrm{mmol} / \mathrm{L}^{*}\end{array}$ & 26 & 46 & 11 & $<0.01$ \\
\hline $\begin{array}{l}\text { Triglycerides } \\
>1.7 \mathrm{mmol} / \mathrm{L}\end{array}$ & 27 & 48 & 13 & $<0.01$ \\
\hline $\begin{array}{l}\mathrm{HDL} \\
\text { (males }<1.03 \mathrm{mmol} / \mathrm{L}) \\
\text { (females }<1.29 \mathrm{mmol} / \mathrm{L})\end{array}$ & 63 & 80 & 50 & 0.01 \\
\hline $\begin{array}{l}\text { Blood pressure } \\
(\geq \mid 30 / \geq 85 \mathrm{mmHg})^{*}\end{array}$ & 52 & 83 & 28 & $<0.01$ \\
\hline
\end{tabular}

"Patients on specific treatment for blood pressure and fasting plasma glucose were included, irrespective of the value obtained.

TABLE 3: Comparison of Cardiovascular Risk Factors between INTERHEART Africa Study and R.K. Khan Hospital Study.

\begin{tabular}{|c|c|c|c|c|}
\hline Characteristics & $\begin{array}{l}\text { INTERHEART Africa \% } \\
(n=\mid 44)\end{array}$ & $\begin{array}{l}\text { R. K. Khan } \\
\%(n=94)\end{array}$ & OR (95\% Cl) & P-value \\
\hline Male & 64 & 62 & $0.91(0.51-1.62)$ & 0.73 \\
\hline Hypertension & 50 & 73 & $2.76(1.52-5.06)$ & $<0.01$ \\
\hline Diabetes & 24 & 40 & $2.11(1.16-3.85)$ & $<0.01$ \\
\hline Smoker (current/former) & 45 & 48 & $1.12(0.64-1.94)$ & 0.68 \\
\hline Continuous variables & Mean (SD) & Mean (SD) & \multicolumn{2}{|c|}{ P-value } \\
\hline Age & $52.3(12.3)$ & $54.3(11.0)$ & \multicolumn{2}{|c|}{0.20} \\
\hline Total cholesterol & $4.50(1.18)$ & $4.80(1.74)$ & \multicolumn{2}{|c|}{0.11} \\
\hline HDL cholesterol & $0.99(0.45)$ & $1.03(0.35)$ & \multicolumn{2}{|c|}{0.46} \\
\hline LDL cholesterol & $2.83(0.97)$ & $3.02(1.12)$ & \multicolumn{2}{|c|}{0.16} \\
\hline BMI & $28.4(5.2)$ & $28.1(5.9)$ & \multicolumn{2}{|c|}{0.68} \\
\hline
\end{tabular}

were on highly active antiretroviral therapy (HAART). This presented us with the unique opportunity of studying a HAART- naive population, thus negating the potential effects of HAART on thrombotic risk. Consistent with the study by Becker, et al. ${ }^{(20)}$ our treatment-naïve HIV positive patients with $\mathrm{AMI}$ presented at a young age and had a high prevalence of smoking. In contrast, however, we did not find any significant difference in traditional cardiovascular risk factors between HIV positive and HIV negative patients. In addition, we found no significant difference in MACE between the 2 groups.

The metabolic syndrome contributes to an increased risk of cardiovascular disease. (21) Many of the metabolic abnormalities common to this syndrome such as insulin resistance, central obesity, dyslipidaemia and hypertension are all independent risk factors for the development of cardiovascular disease, which when grouped together, amplify this risk. It is also well recognised that the incidence of the metabolic syndrome varies among different ethnic groups. ${ }^{(22)}$ The prevalence of the meta- bolic syndrome in Asian Indians presenting with AMI to the CCU at R.K. Khan Hospital was reported to be $57 \%$ according to the IDF criteria. ${ }^{(23)}$

Similarly, in Black patients with AMI, the metabolic syndrome was seen in $45 \%$ of the study population based on the IDF definition. These results concur with another study amongst South African Blacks with CAD in whom the metabolic syndrome was found to be highly prevalent (60\%).(II) The metabolic syndrome, therefore, may be another factor contributing to the increase in AMI in the Black population.

More importantly, following multivariable analysis using logistic regression, our data showed that several clinical and laboratory parameters are significantly associated with MACE such as an increased heart rate $(p=0.01)$ STEMI $(p=0.03)$, obesity $(p=0.03)$, a family history of cerebrovascular disease $(p=0.04)$ and hyperuricaemia $(p=0.04)$. 


\section{LIMITATIONS}

Potential limitations of this study merit consideration. Firstly, because of the relatively small sample size, results for smaller subgroups should be interpreted with caution. Secondly, this was a single centre study and selection bias might exist. Finally, not all patients were subjected to cardiac catheterisation studies and data on angiographic severity of CAD and cardiac revascularisation was, therefore, tentative.

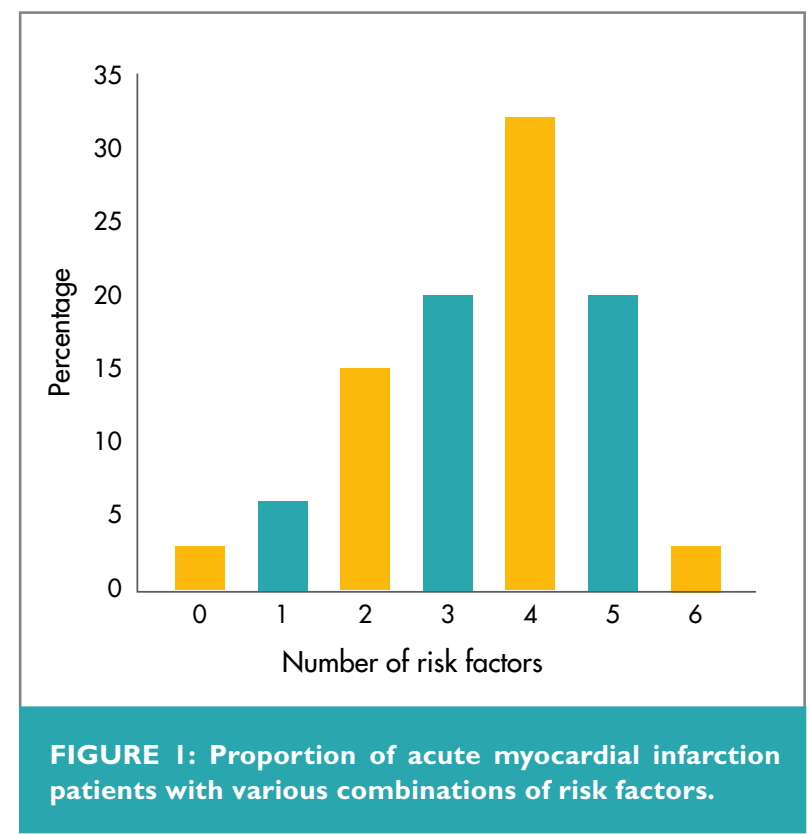

Includes any combination of diabetes, smoking, family history of vascular disease dyslipidaemia, hypertension and visceral obesity.

\section{CONCLUSION}

This study demonstrates that South African Blacks have multiple risk factors, similar to other ethnic groups in the country, which contribute to the development of AMI. In addition to conventional risk factors for CAD, an increased heart rate, STEMI, obesity, a family history of cerebrovascular disease and hyperuricarmia are strongly associated with the presence of MACE. Furthermore, recurrence of infarction and death occurred more frequently in females compared to males. These results highlight the urgent need for lifestyle modification and effective treatment of CAD risk factors, particularly in the Black population.

\section{ACKNOWLEDGEMENTS}

We thank Ms S. Ramdas for the typing of this manuscript.

\section{Conflict of interest: none declared.}

TABLE 4: Bivariate and multivariate logistic regression analysis of clinical and laboratory parameters associated with MACE.

\begin{tabular}{|c|c|c|c|c|}
\hline Characteristics & $\begin{array}{c}\text { Bivariate } \\
\text { OR }(95 \% \mathrm{Cl})\end{array}$ & P-value & $\begin{array}{l}\text { Multivariable } \\
\text { OR }(95 \% \mathrm{CI})\end{array}$ & P-value \\
\hline Age & $1.03(0.99-1.08)$ & 0.11 & $1.02(0.96-1.08)$ & 0.55 \\
\hline Male & $0.56(0.24-1.33)$ & 0.19 & $0.72(0.14-3.56)$ & 0.68 \\
\hline Heart rate & $1.03(1.01-1.06)$ & 0.02 & $1.06(1.01-1.10)$ & 0.01 \\
\hline HIV positive & $0.69(0.22-2.16)$ & 0.52 & $0.65(0.13-3.15)$ & 0.59 \\
\hline STEMI vs. NSTEMI & $2.0(0.67-5.93)$ & 0.21 & $5.98(1.22-28.5 \mathrm{I})$ & 0.03 \\
\hline Diabetes & $1.54(0.65-3.61)$ & 0.33 & $1.03(0.27-3.89)$ & 0.97 \\
\hline Hypertension & $1.01(0.39-2.62)$ & 0.98 & $0.95(0.23-3.93)$ & 0.95 \\
\hline Smoking & $0.54(0.23-1.28)$ & 0.16 & $0.60(0.13-2.78)$ & 0.51 \\
\hline Obesity & $1.05(0.43-2.56)$ & 0.92 & $3.86(1.18-12.63)$ & 0.03 \\
\hline Family history of cerebrovascular disease & $7.87(0.84-73.52)$ & 0.07 & $14.83(1.10-200.82)$ & 0.04 \\
\hline Metabolic syndrome & $0.86(0.34-2.19)$ & 0.75 & $0.66(0.18-2.43)$ & 0.53 \\
\hline Creatinine & $1.02(1.0-1.04)$ & 0.04 & $1.02(0.99-1.04)$ & 0.13 \\
\hline Blood glucose & $1.06(0.99-1.14)$ & 0.11 & $1.0(0.88-1.12)$ & 0.94 \\
\hline Uric acid & $2.53(1.05-6.07)$ & 0.04 & $3.64(1.05-12.66)$ & 0.04 \\
\hline Triglycerides & $0.86(0.59-1.25)$ & 0.42 & $0.82(0.48-1.40)$ & 0.47 \\
\hline
\end{tabular}




\section{REFERENCES}

I. Derry CW, Bourne DE, Sayed AR, et al. Variations in mortality of the Coloured, White and Asian population groups in the RSA, 1978-1982. Part VI. Ischaemic heart disease. S Afr Med J 1987;72:698-70.

2. Ranjith N, Pegoraro RJ, Zaahl MG. Risk factors associated with acute coronary syndromes in South African Asian Indian patients (The AIR Study). J Clin Exp Cardio 201 1;2:163-7.

3. Ranjith N, Pegoraro RJ, Naidoo DP. Demographic data and outcome of acute coronary syndrome in South African Asian Indian population. Cardiovasc J S Afr 2005; 16:48-54. Epub 2004 Nov 30.

4. Seedat YK, Mayet FG, Latiff GH, et al. Risk factors and coronary heart disease in Durban Blacks-the missing links. S Afr Med J 1992;82:25 I-6.

5. Seftel HC, Asvat MS, Joffe Bl, et al. Selected risk factors for coronary heart disease in male scholars from the major South African population groups. S Afr Med J 1993;83: 89|-7.

6. Steyn K, Sliwa K, Hawken S, et al. Risk factors associated with myocardial infarction in African. The INTERHEART Africa Study. Circ 2005;1 12: 3554-61.

7. Yusuf S, Hawken S, Ounpuu S. Effect of potentially modifiable risk factors associated with myocardial infarction in 52 countries (the INTERHEART Study): Case-control study. Lanc 2004;364:937-52

8. Amira C, Ntyintyane L, Wilkinson D, et al. Emerging epidemic of cardiovascular disease among urban Africans: African coronary syndrome at Baragwanath Hospital, Soweto. SA H Spr 2006:7-1 I.

9. UNAIDS Sub-Saharan Africa. Aids epidemic update. Regional Summary 2008. Accessed March 2009 http://data.unaids.org/pub/Report/2008/jc 1526_epibriefs_ssafrican_en.pdf.

10. Lorenz MW, Stephan C, Harmjanz A, et al. Both long-term HIV infection and highly active antiretroviral therapy are independent risk factors for early carotid atherosclerosis. Atherosc 2008;196:720-6.

II. Ntyintyane LM, Panz VR, Raal FJ, et al. Metabolic syndrome, undiagnosed diabetes mellitus and insulin resistance are highly prevalent in urbanised South African blacks with coronary artery disease. Cardio J S Africa 2006; 17:50-5.

12. The Joint European Society of Cardiology/American College of Cardiology Committee. Myocardial infarction redefined-a consensus document of the Joint European Society of Cardiology/American College of Cardiology Committee for the redefinition of myocardial infarction. J Am Coll Cardio 2000;36:959-69.

13. World Health Organisation (1998) Obesity: Preventing and managing the global epidemic. Report of a WHO Consultation on Obesity, Geneva, 3-5 June, 1997. Geneva, Switzerland: World Health Organisation (WHO TRS 894)

14. International Diabetes Federation. The IDF Consensus Worldwide Definition of the Metabolic Syndrome. April 14, 2005: (accessed June 10, 2005) http://www.idf.org/webdata/docs/Metac_syndrome_def.pdf

15. Yusuf S, Reddy S, Ounpuu S, et al. Global burden of cardiovascular disease, part II: Variations in cardiovascular disease by specific ethnic groups and geographic regions and prevention strategies. Circ 200 I; 104:2855-64.

16. Annual Report of the Medical Officer of Health. City of Johannesburg, South Africa 1994.

17. Chesler E, Mitha AS, Weir EK et al. Myocardial infarction in the black population of South Africa: Coronary arteriographic finding. Am Heart J 1978;95:691-6.

18. Fuchs SC, Alencastro PR, Leticia MR, et al. Risk of coronary heart disease among HIV - infected patients: A multicenter study in Brazil. The Scientific World Journal, 2013;1-8. Epub 2013 Oct 2
19. Mondy K, Overton ET, Grubb J, et al. Metabolic syndrome in HIV- infected patients from an urban, Midwestern US outpatient population, Clin Inf Disease 2007:4:726-34

20. Becker AC, Jacobson B, Singh $\mathrm{S}$, et al. The thrombotic profile of treatmentnaïve HIV positive Black South Africans with acute coronary syndromes, Clin Appl Thromb Hemost, 2010;1 -9.

21. Malik S, Wong ND, Franklin S, et al. Impact of the metabolic syndrome on mortality from coronary heart disease, cardiovascular disease and all causes in United States adults. Circ 2004; I 1 0: 1245-50.

22. Ford ES, Giles $\mathrm{WH}$, Dietz $\mathrm{WH}$. Prevalence of the metabolic syndrome among US adults: Findings from the third National Health and Nutrition Examination Survey. J Am Med Assoc 2002;287:356-9.

23. Ranjith N, Pegoraro RJ, Naidoo DP, et al. Metabolic syndrome in young Asian Indian patients with myocardial infarction. Cardio J S Africa 2007: | 8:2|-6. 